\title{
Efficient Methods for FFT calculations Using Memory Reduction Techniques.
}

\author{
N. Kalaiarasi \\ Assistant professor \\ SRM University \\ Kattankulathur, chennai
}

\author{
A.Rathinam \\ Assistant professor \\ SRM University \\ Kattankulathur,chennai
}

\begin{abstract}
FFT algorithms is one of the many methods used for the calculation of DFT, but they are preferred due to their increased speed and higher efficiency, which arises due to the fact that for the calculation of a N-point DFT, the sequence is broken into several segments and the DFT for each segment is calculated. However, for this many redundant memory spaces are required. The Butterfly structure for the calculation of DFT by FFT algorithms is preferred due to its symmetry which makes it suitable for hardware implementations, but this requires the loading of the twiddle factors for each stage repeatedly, which leads to inefficient use of memory space. To overcome this, grouping the identical twiddle factors of different stages together reduces the number of memory references and the storage space due to twiddle factors, therein reducing the number of clock cycles needed for the complete implementation of the algorithm. Thus this can be an efficient method for the calculation of FFT algorithms.

The Decimation In Time (DIT) and Decimation In Frequency (DIF) algorithms can be implemented in MATLAB- Simulink blocks to verify their accuracy and coded in $\mathrm{C}$ language to verify their reduction in computation time. The same can also be verified using Code composer studio (CCS) and implemented in DSP Processors for the verification of reduced clock cycles. The reduction in memory spaces and memory references of the twiddle factors is evident implicitly when compared with conventional methods.
\end{abstract}

The full text of the article is not available in the cache. Kindly refer the IJCA digital library at www.ijcaonline.org for the complete article. In case, you face problems while downloading the full-text, please send a mail to editor at editor@ijcaonline.org 